\title{
Therapeutic targeting of liver cancer with a recombinant DNA vaccine containing the hemagglutinin-neuraminidase gene of Newcastle disease virus via apoptotic-dependent pathways
}

\author{
LI-GANG CHEN* ${ }^{*}$ YUAN-SHENG LIU* , TANG-HUI ZHENG, XU CHEN, \\ PING LI, CHUAN-XING XIAO and JIAN-LIN REN
}

Department of Gastroenterology, Zhongshan Hospital Affiliated to Xiamen University, Xiamen, Fujian 361004, P.R. China

Received April 9, 2015; Accepted August 9, 2016

DOI: $10.3892 / \mathrm{ol} .2016 .5114$

\begin{abstract}
A total of 38.6 million mortalities occur due to liver cancer annually, worldwide. Although a variety of therapeutic methods are available, the efficacy of treatment at present is extremely limited due to an increased risk of malignancy and inherently poor prognosis of liver cancer. Gene therapy is considered a promising option, and has shown notable potential for the comprehensive therapy of liver cancer, in keeping with advances that have been made in the development of cancer molecular biology. The present study aimed to investigate the synergistic effects of the abilities of the hemagglutinin neuraminidase protein of Newcastle disease virus (NDV), the pro-apoptotic factor apoptin from chicken anaemia virus, and the interferon- $\gamma$ inducer interleukin-18 (IL-18) in antagonizing liver cancer. Therefore, a recombinant DNA plasmid expressing the three exogenous genes, $V P 3, I L-18$ and hemagglutinin neuraminidase $(H N)$, was constructed. Flow cytometry, acridine orange/ethidium bromide staining and analysis of caspase- 3 activity were performed in $\mathrm{H} 22$ cell lines transfected with the recombinant DNA plasmid. In addition, 6-week-old C57BL/6 mice were used to establish a $\mathrm{H} 22$ hepatoma-bearing mouse model. Mice tumor tissue was analyzed by immunohistochemistry and scanning electron microscopy. The results of the present study revealed that the recombinant DNA vaccine containing the VP3, IL- 18 and $H N$ genes inhibited cell proliferation and induced autophagy via the mitochondrial pathway in vivo and in vitro.
\end{abstract}

Correspondence to: Dr Chuan-Xing Xiao or Professor Li-Gang Chen, Department of Gastroenterology, Zhongshan Hospital Affiliated to Xiamen University, 201 Hubin South Road, Xiamen, Fujian 361004, P.R. China

E-mail: xiaoxx@163.com

E-mail: chenligang663@163.com

*Contributed equally

Key words: Newcastle disease virus, hemagglutinin neuraminidase gene, recombinant plasmid, gene therapy, hepatocellular carcinoma, apoptosis

\section{Introduction}

Gene therapy for liver cancer remains a rapidly progressing field $(1,2)$. For example, the p53 gene plays a pivotal role in gene therapy for liver cancer. Irrespective of whether the p53 gene was mutated, wild-type or heterozygous in the cells of interest, tumor cell growth was suppressed subsequent to introducing an exogenous wild-type p53 gene $(3,4)$. It was subsequently found that increased p53 protein expression levels could markedly suppress tumor cell growth. In addition, suicide genes have been extensively utilized in the setting of liver cancer gene therapy (5).

Previously, a large number of targeted genes have been selected for cancer gene therapy, and gene transfer methods have also been advanced during this time (6). Newcastle disease virus (NDV) is a member of the Paramyxoviridae family whose genome is a non-segmented single-stranded negative-sense RNA (7). The virus genome encodes 6 genes in the following order $\left(3^{\prime}-5^{\prime}\right)$ : Nucleoprotein $(\mathrm{NP})$; phosphoprotein $(\mathrm{P})$; matrix protein (M); fusion protein $(\mathrm{F})$; hemagglutinin neuraminidase $(\mathrm{HN})$; and large polymerase protein (L) (3'-NP-P-M-F-HN-L-5') $(8,9)$. It was previously shown that the $\mathrm{HN}$ protein plays a critical role in the anti-tumor effects of NDV (10). HN hydrolyzes the surface sialic acid of the host cell, exposes biological recognition sites, and induces tumor necrosis factor-associated apoptosis-inducing ligand (TRAIL) expression at the surface of mononuclear cells in the host peripheral blood, and yet does so independently of viral replication $(11,12)$. In addition, HN positioning in the tumor cell membrane also led to formation of identical recognition sites, which in turn improved the cytotoxic effects of the host immune system against tumor cells (13).

Apoptin is a small protein that is derived from chicken anemia virus. It can only induce apoptosis in transformed or tumorigenic cells, rather than primary cells (14). Apoptin-induced apoptosis of tumor cells is independent of functional p53 expression, and occurs in the presence of high Bcl-2 expression levels. A DNA vaccine containing the VP3 gene can activate specific cytotoxic T lymphocytes (CTLs) and T-helper cells, and humoral immune responses (15).

Interleukin (IL)-18 has similar biological activities as those reported for IL-12, yet is more powerful than IL-12 at inducing interferon (IFN) $-\gamma$ (16). Thus, it is also termed IFN- $\gamma$ inducing 
factor. IL-18 is a cytokine with pleiotropic biological activities in terms of promoting dendritic cell maturation, stimulating NK and CTL cell-mediated cytotoxicity, inducing secretion of cytokines such as IFN, IL-2 and granulocyte-macrophage colony-stimulating factor (GM-CSF), and subsequent expression of major histocompatibility complex class I molecules (16). Therefore, IL-18 can be used as an adjuvant to enhance the immunological effects of specific candidate vaccines.

In the present study, with respect to the identical positioning of the NDV HN protein at the membrane surface subsequent to expression in tumor cells, NDV HN was employed as a tumor-specific antigen in the form of a DNA vaccine to improve host immunity, thereby strengthening the immunological clearance of tumor cells carrying this protein. As an IFN- $\gamma$ inducer, IL-18 promotes anti-tumor effects by enhancing the activity of NK and T cells. In this context, a recombinant DNA plasmid was constructed to co-express HN, VP3 and IL-18, and the mechanisms responsible for the anti-tumor effects was investigated both in vitro and in vivo.

\section{Materials and methods}

Ethics. All procedures involving animals were performed in accordance with protocols that were approved by the Committee for Animal Research of Xiamen University (Xiamen, Fujian, China) and complied with the Guide for the Care and Use of Laboratory Animals (17).

Plasmids, cell culture and transfection. The recombinant plasmids pIRESneo (Invitrogen; Thermo Fisher Scientific, Inc., Waltham, MA, USA), pIRHN, PIRVP3 and pIRVP3IL-18HN were constructed and validated at the Department of Gastroenterology, Zhongshan Hospital Affiliated to Xiamen University (Xiamen, China). Briefly, the pIRESneo plasmid was spliced by $S m a \mathrm{I}$ and $\mathrm{XbaI}$ restriction enzymes (Takara Bio, Inc., Otsu, Japan) and transfected with HN, VP3 and VP3IL-18HN gene fragments (BGI, Shenzhen, China) using T4 DNA ligase (Takara Bio, Inc.) to obtain pIRHN, PIRVP3 and pIRVP3IL-18HN plasmids, respectively. Mouse H22 hepatoma cells (Experimental Animal Centre of Jilin University, Changchun, Jilin, China) were cultured in Dulbecco's modified Eagle medium containing $10 \%$ fetal bovine serum, $100 \mathrm{U} / 1$ penicillin, and $100 \mathrm{U} / 1$ streptomycin (Gibco; Thermo Fisher Scientific, Inc.) at $37^{\circ} \mathrm{C}$ in an atmosphere of $\mathrm{CO}_{2}$. Cell lines that stably expressed HN, VP3 and IL-18 were established by transducing H22 cells with the pIRVP3IL-18HN lentiviral vector.

Western blot analysis. H22 cells were transfected with pIRVP3IL-18HN and pIRESneo using Lipofectamine 2000 (Invitrogen; ThermoFisherScientific,Inc.)for $24 \mathrm{~h}$.Subsequently, $2 \times 10^{5}$ transfected $\mathrm{H} 22$ cells were lysed in radioimmunoprecipitation assay buffer consisting of $50 \mathrm{mmol} / 1$ Tris ( $\mathrm{pH} 8.0$ ), $0.1 \%$ sodium dodecyl sulfate, $0.5 \%$ sodium deoxycholate, $1 \%$ NP-40, $150 \mathrm{mmol} / 1 \mathrm{NaCl}$, and 1 tablet of complete mini protease inhibitor/10 ml of buffer (Roche Diagnostics GmbH, Penzberg, Germany). Equal amounts of total cell lysate were electrophoresed and transferred to FluoroTrans ${ }^{\circledR} \mathrm{W}$ membranes (Wako Pure Chemical Industries Ltd., Wako, Japan). The membranes were then incubated with monoclonal goat anti-mouse Flag antibody (cat. no. 66008-2-Ig; 1:1,000; Proteintech Group, Inc.,
Rosemont, IL, USA). Subsequent to washing, the membrane was incubated with horseradish peroxidase-conjugated goat anti-rabbit IgG secondary antibody (cat. no. SA00001-2; 1:5,000; Proteintech Group, Inc.) and visualized by an enhanced chemiluminescence detection system (ECL Advance; GE Healthcare Life Sciences, Chalfont, UK).

Flow cytometry. H22 cells were transfected with pIRVP3IL-18HN and pIRESneo for $24 \mathrm{~h}$, following which $5 \times 10^{5}$ cells were stained using fluorescein isothiocyanate (FITC)-labeled monoclonal goat anti-mouse HLA-A, B and C antibodies (cat. no. ab23840; 1:100; Abcam, Cambridge, MA, USA) and Rhodamine 123 at a final concentration of $25 \mathrm{mg} / \mathrm{ml}$. Following antibody incubation for $1 \mathrm{~h}$ at $4^{\circ} \mathrm{C}$ in the dark, the cells were washed and treated with fluorescence-activated cell sorting lysing solution, according to the manufacturer's protocol (Becton Dickinson, Franklin Lakes, NJ, USA). In each sample, $10^{4}$ cells were counted by multi-parameter flow cytometry (FACScan; Becton Dickinson) and analyzed by CellQuest 5.1 software (Becton Dickinson).

2,7-Dichlorofluorescin diacetate (DCFA) analyses. DCFA analyses were performed to assess reactive oxygen species activation. H22 cells were transfected with pIRESneo and pIRVP3IL $18 \mathrm{HN}$. After incubation for $72 \mathrm{~h}$, a total of $2 \times 10^{4} \mathrm{HCC}$ cells were harvested and stained with $5 \mu \mathrm{mol} / 1 \mathrm{DCFA}$ at $37^{\circ} \mathrm{C}$ for $30 \mathrm{~min}$ in the dark. Cells were then washed twice with phosphate-buffered saline (PBS)and counted by multi parameter flow cytometry (FACScan; Becton Dickinson) and analyzed by CellQuest 5.1 software as described above.

Proliferation assay. $\mathrm{H} 22$ cells were transfected with the pIRVP3IL-18HN and pIRESneo plasmids for $24 \mathrm{~h}$. The cells were seeded at a density of 3,000 cells per well into 96-well plates and incubated at $37^{\circ} \mathrm{C}$ overnight. The next day, the media was aspirated and the cells were washed twice in PBS and then starved for $24 \mathrm{~h}$ in a serum-free medium containing $0.1 \%$ bovine serum albumin. Methyl thiazolyl tetrazolium reagent (Sigma-Aldrich; Merck Millipore, Darmstadt, Germany) was added to each well, cells were lysed with dimethyl sulfoxide and then quantified by measuring the absorbance at A570 nm using an enzyme-linked immunosorbent assay plate reader (Bio-Rad Laboratories, Inc., Hercules, CA, USA). Each treatment was conducted in triplicate, and all experiments were conducted independently at least three times.

Acridine orange $(A O)$ /ethidium bromide $(E B)$ staining. In total, $25 \mu \mathrm{l}$ of a suspension of cells $\left(0.5-2.0 \times 10^{6}\right)$ were mixed gently with $1 \mu \mathrm{l}$ of $\mathrm{AO} / \mathrm{EB}$ solution, which was a mixture of $100 \mu \mathrm{g} / \mathrm{ml} \mathrm{AO}$ in PBS and $100 \mu \mathrm{g} / \mathrm{ml}$ EB in PBS. Subsequently, $10 \mu \mathrm{l}$ of stained cell suspension was loaded onto a slide and $\geq 300$ cells were counted under a fluorescence microscope that was equipped with a fluorescence filter and an objective lens of x40 magnification.

Caspase-3 activity analysis. In total, $\sim 5 \times 10^{6}$ cells were resuspended in $200 \mu \mathrm{l}$ lysis buffer (HD Biosciences Co., Ltd., Shanghai, China) consisting of $20 \mathrm{mmol} / 1$ piperazine-N,N'-bis(2-ethanesulfonic acid) (pH 7.2), $100 \mathrm{mmol} / \mathrm{l}$ $\mathrm{NaCl}, 1 \mathrm{mmol} / \mathrm{l}$ ethylenediaminetetraacetic acid (EDTA; HD 
Biosciences Co.,Ltd.), $10 \mathrm{mmol} / \mathrm{l}$ dithiothreitol (DTT) (Solarbio Science \& Technology Co.,Ltd., Beijing, China), $0.1 \%$ 3-[(3-cholamidopropyl) dimethylammonio]-1-propanesulfonate (HD Biosciences Co., Ltd.) and 10\% sucrose (HD Biosciences Co., Ltd.) and incubated on ice prior to centrifugation at 3,000 $\mathrm{x}$ for $5 \mathrm{~min}$. The supernatant was harvested for protein quantification and caspase- 3 activity was measured using a caspase- 3 assay kit (Abcam) according to the manufacturer's instructions, at an absorbance of $405 \mathrm{~nm}$ using a microplate analyzer.

Extraction of cytochrome $c$. Following transfection with the pIRVP3IL-18HN recombinant plasmid for $72 \mathrm{~h}, 2 \times 10^{6} \mathrm{H} 22$ tumor cells were lysed in $300 \mu$ lof buffer A, consisting of $10 \mathrm{mM}$ 4-(2-hydroxyethyl)-1-piperazineethanesulfonic acid- $\mathrm{KOH}$ ( $\mathrm{pH}$ 7.4), $0.25 \mathrm{M}$ sucrose, $1 \mathrm{mM}$ ethylene glycol-bis( $\beta$-aminoethyl ether)-N,N,N',N'-tetraacetic acid, $1 \mathrm{mM}$ EDTA, $1 \mathrm{mM}$ DTT, $10 \mathrm{mM} \mathrm{MgCl}{ }_{2}$ and $1 \mathrm{mM}$ phenylmethylsulfonyl fluoride (all obtained from Solarbio Science \& Technology Co., Ltd.) and homogenized in a Dounce homogenizer (IKA, Guangzhou, China) for $10 \mathrm{~min}$. The supernatant was mixed with TNC buffer, consisting of $10 \mathrm{mM}$ Tris-acetate (pH 8.0) (HD Biosciences Co., Ltd.), 0.5\% Nonidet P-40 (HD Biosciences Co., Ltd.) and $5 \mathrm{mM} \mathrm{CaCl}_{2}$ (Solarbio Science \& Technology Co., Ltd.), and the precipitate was dissolved in buffer A to obtain the cytosol, which together with the mitochondria were stored at $-80^{\circ} \mathrm{C}$ for subsequent western blot analysis.

Establishment of H22 hepatoma-bearing C57BL/6 mouse model. Six-week-old C57BL/6 male mice $(\mathrm{n}=15)$ were obtained from the Experimental Animal Center of the Academy of Military Medical Sciences of the Chinese People's Liberation Army (license number: SCXK-[Army] 2002-001; Beijing, China). The mice were used to establish the H22-bearing mouse model. H22 cells in the logarithmic growth phase were adoptively transferred subcutaneously into the right hind foot of mice at a dose of $0.1 \mathrm{ml}$ each. When the tumors had grown to $>5 \mathrm{~mm}$ in diameter, the tumor-bearing mice were randomly divided into 5 groups as follows: PBS control group; blank plasmid pIRESneo-treated group; pIRHN-treated group; pIRVP3-treated group; and pIRVP3IL-18HN-treated group. An extra group of wild-type mice were included as normal controls. All groups were inoculated once every 7 days, a total of 3 times, at a dose of $100 \mu \mathrm{l} /$ mouse with PBS or plasmids that were diluted in PBS to a concentration of $50 \mu \mathrm{g} / \mathrm{ml}$ ( $5 \mu \mathrm{g}$ plasmids/mouse). All mice were sacrificed on day 7 subsequent to the last injection and were evaluated for various markers.

Scanning electron microscopy analysis. Animals were sacrificed on day 7 subsequent to the last injection and the tumors were removed and quickly fixed in ice-cold glutaraldehyde (Sigma-Aldrich; Thermo Fisher Scientific, Inc.). Subsequently, the tumor was sectioned into $1 \mathrm{~mm}^{3}$ blocks, and then fixed for another $12 \mathrm{~h}$ in cold glutaraldehyde at $4^{\circ} \mathrm{C}$. Following three washes in $0.13 \mathrm{M}$ phosphate buffer, the tissue blocks were fixed with ice-cold $1 \%$ osmium tetroxide (Sinopharm Chemical Reagent Co., Ltd., Shanghai, China) for 1.5-2 h, followed by sequential $15 \mathrm{~min}$ incubations in 50,70 and $80 \%$ ethanol at $4^{\circ} \mathrm{C}$. Subsequently, room temperature fixing steps in 90 and $100 \%$ ethanol and a final acetone step at room temperature were performed. The blocks were gradually permeabilized in a series of buffers that consisted of dehydrating agents mixed with epoxy resin at a ratio of 2:1, 1:1 and 1:3, followed by $100 \%$ resin, for $0.5-1 \mathrm{~h}$ each incubation. The blocks were then placed into labeled capsules containing embedding medium and polymerized at 35,45 and $60^{\circ} \mathrm{C}$ for 12,12 and $24 \mathrm{~h}$, respectively, in an incubator. The polymerized tissue blocks were shaped and dissected into slices at a thickness of 600-700 A. The slices were placed on a wax plate covered by a copper mesh and stained with uranyl acetate dye and lead citrate dye for 15-20 and 5-10 min, respectively, followed by distilled water washes and examination by electron microscopy.

Immunohistochemistry. The mice were sacrificed and the tumors were instantly removed and incubated in $10 \%$ neutral formalin for $24-72 \mathrm{~h}$ for fixing. The formalin was discarded and the tumor tissue was treated sequentially with $60,70,80,95$ and $100 \%$ ethanol, $50 \%$ ethanol plus 50\% xylene, and xylene. Subsequently, the tissue was embedded in a paraffin block and sectioned into slices at 4-6 $\mu \mathrm{m}$ thickness, which were then incubated in a $45^{\circ} \mathrm{C}$ water bath and transferred onto glass slides, and then covered with protein glycerin prior to dehydrating in an oven at $60^{\circ} \mathrm{C}$ for $2 \mathrm{~h}$. Subsequent to routine hematoxylin-eosin staining, the slices were mounted with mounting medium and examined under a standard light microscope (x10 and x40 magnifcation).

Statistical analysis. Data were expressed as the mean \pm standard deviation. Groups were compared using Student's $t$-test, and values of $\mathrm{P}<0.05$ were considered to indicate a statistically significant difference.

\section{Results}

Inhibition of liver cancer cell growth via pro-apoptotic pathways in vitro by a recombinant DNA vaccine containing the NDV HN gene. $\mathrm{H} 22$ hepatoma cells were transfected with the newly constructed DNA vaccine pIRVP3IL-18HN, to validate its function against liver cancer (Fig. 1A). It was found that the cytotoxic effects of pIRVP3IL-18HN on H22 hepatoma cells were positively associated with time and DNA concentrations, and ultimately peaked $72 \mathrm{~h}$ subsequent to transfection (Fig. 1B). As observed under optical microscopy, $\mathrm{H} 22$ hepatoma cells that were transfected with pIRVP3IL-18HN detached from the plate after $72 \mathrm{~h}$, which was in contrast to those transfected with the control plasmid pIRESneo (Fig. 1C). Additional examination by electron microscopy visualized chromatin margination and condensation in transfected $\mathrm{H} 22$ cells, which were typical apoptotic features, and known as pyknotic nuclei. By contrast, tumor cells transfected with the control plasmid exhibited relatively normal morphology and nuclear structures (Fig. 1C). Upon $\mathrm{AO} / \mathrm{EB}$ staining, the control cells were presented as ubiquitously fluorescent green cells.

However, at $72 \mathrm{~h}$ subsequent to transfection with pIRVP3IL-18HN in vitro, certain H22 tumor cells were exhibited as bright orange cells with chromatin condensation. In addition, certain cells were fluorescent red following a general loss of cell membrane integrity in combination with reduced sizes of the nuclei, and particularly so in the later stage of apoptosis (Fig. D). In addition, cell cycle changes were observed in $\mathrm{H} 22$ cells, as detected by propidium iodide staining followed by 
A

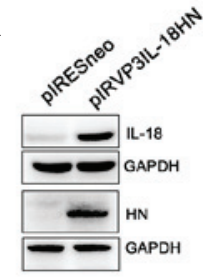

C
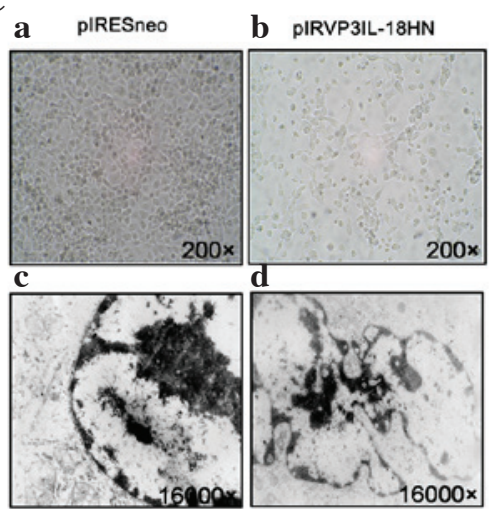

$\mathbf{E}$

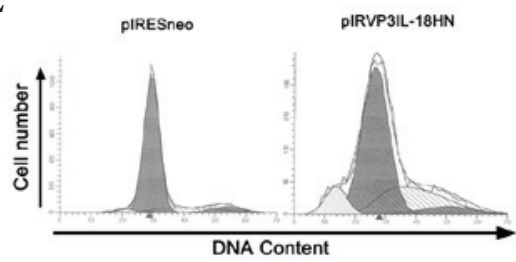

B
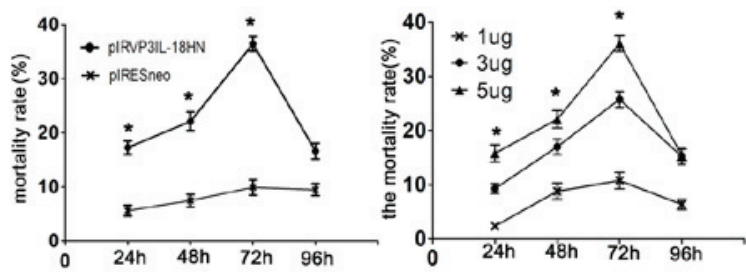

D
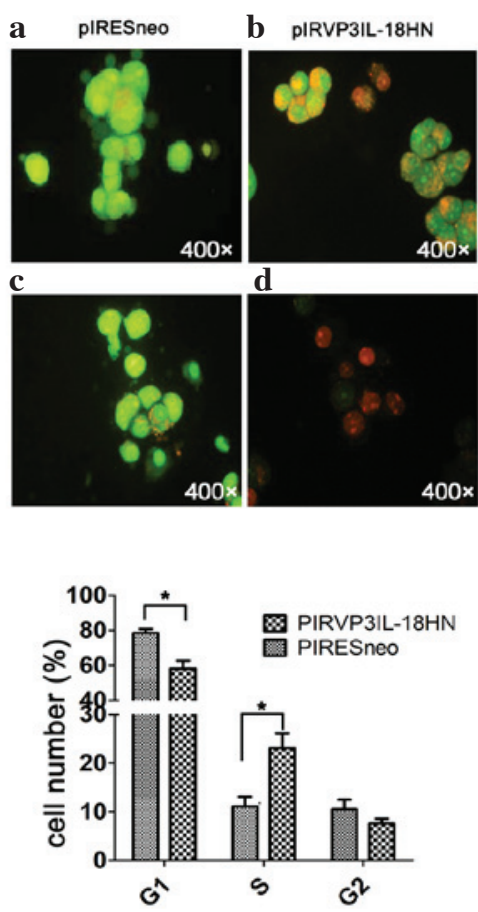

Figure 1. The pIRVP3IL-18HN recombinant plasmid promoted apoptosis of $\mathrm{H} 22$ hepatoma cells. (A) Western blot analysis of the expression of pIRVP3IL-18HN in hepatoma cells. (B) Cytotoxic effects of pIRVP3IL-18HN on H22 cells were time- and concentration-dependent. (C) Observation of the apoptotic morphology of $\mathrm{H} 22$ hepatoma cells at $72 \mathrm{~h}$ after transfection with $(\mathrm{Ca})$ pIRESneo and $(\mathrm{Cb})$ pIRVP3IL-18HN plasmids under a light microscope at x200 magnification and by (Cc and $\mathrm{Cd}$ ) transmission electron microscopy at x16,000 magnification. (D) Modifications observed in H22 hepatoma cells that were stained with acridine orange/ethidium bromide as observed under fluorescence microscopy at (Da and Db) 48 and (Dc and Dd) $72 \mathrm{~h}$ after transfection with pIRESneo and pIRVP3IL-18HN. (E) Flow cytometric analysis of H22 cell cycle at $72 \mathrm{~h}$ after transfection with pIRESneo and pIRVP3IL-18HN revealed that pIRVP3IL-18HN arrested the cell cycle at S phase. "P<0.05. IL-18, interleukin 18; GAPDH, glyceraldehyde 3-phosphate dehydrogenase; HN, hemagglutinin neuraminidase.

flow cytometry. An apoptotic peak appeared in the G1 phase of the cell cycle with increasing numbers of $\mathrm{H} 22$ cells that were arrested at the $\mathrm{S}$ phase of the cell cycle, which led to an apoptosis rate of $11 \%$ (Fig. 1E).

Apoptosis of tumor cells was possibly induced via the mitochondrial pathway by the recombinant DNA vaccine containing the NDV HN gene. The present study found that, compared with cells carrying the control empty plasmid, mitochondrial uptake of Rhodamine 123 was substantially decreased in H22 tumor cells that had been transfected with pIRVP3IL-18HN in vitro, leading to a peak shift from right to left in flow cytometric analysis, which indicates a change in cell number. This result indicates that pIRVP3IL-18HN could downregulate the mitochondrial membrane potential in $\mathrm{H} 22$ cells, as an early event of apoptosis (Fig. 2A). Additional analysis by DCFA combined with flow cytometry revealed that intracellular levels of reactive oxygen species (ROS) were elevated in $\mathrm{H} 22$ cells following transfection with pIRVP3IL-18HN compared with the control cells, as indicated by a peak shift to the right (Fig. 2B). Enhanced ROS production led to increased apoptosis of tumor cells. Consequently, there were markedly increased levels of cytochrome $c$ detected in pIRVP3IL-18HN-transfected H22 cells compared with control cells (Fig. 2C). The caspase proteases play a critical role in proteolysis and activation of proteins to coordinate the central events that drive apoptosis. The present study analyzed caspase-3 activity in $\mathrm{H} 22$ cells that were transfected with pIRESneo and pIRVP3IL-18HN plasmids. According to the optical density values, caspase- 3 was activated in $\mathrm{H} 22$ cells at $72 \mathrm{~h}$ after transfection with the pIRVP3IL-18HN plasmid, but not in cells transfected with the control plasmid.

Inhibitory effects of the NDV HN recombinant DNA vaccine on liver cancer growth in vivo. A tumor-bearing C57BL/6 mouse model was established using H22 cells (Fig. 3). Compared to the PBS group and the empty plasmid treatment group, treatment with the recombinant plasmid containing individual pIRHN or pIRVP3 genes inhibited H22 tumor growth. Co-expression of 
A

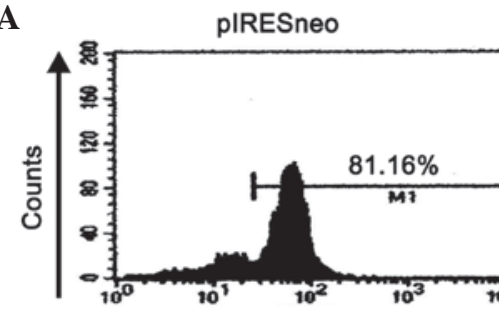

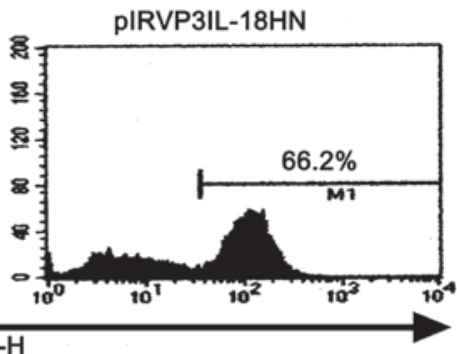

FL1-H

B

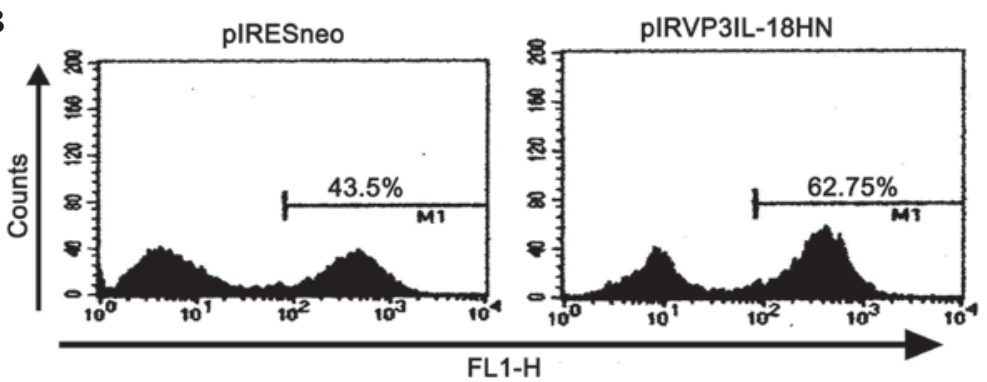

C

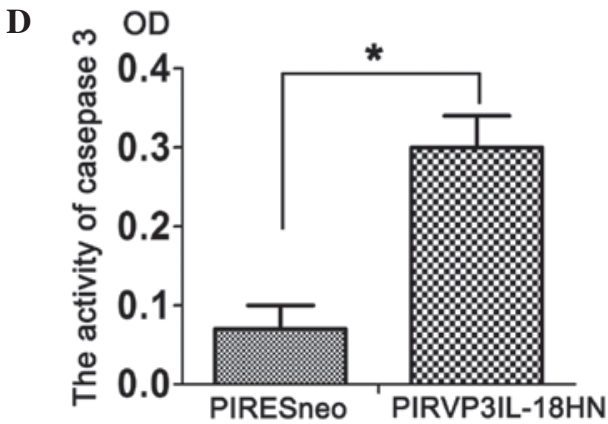

Figure 2. pIRVP3IL-18HN recombinant plasmid promoted apoptosis of H22 cells via the mitochondrial pathway. (A) Assessment of mitochondrial membrane potential in $\mathrm{H} 22$ cells by flow cytometry at $72 \mathrm{~h}$ after transfection with the pIRESneo and pIRVP3IL-18HN plasmids revealed that pIRVP3IL-18HN downregulated the mitochondrial membrane potential in $\mathrm{H} 22$ cells. (B) Measurement of reactive oxygen species in $\mathrm{H} 22$ cells by flow cytometry at $72 \mathrm{~h}$ after transfection with pIRESneo and pIRVP3IL-18HN plasmids demonstrated that pIRVP3IL-18HN enhanced ROS production leading to increased tumor cell apoptosis. (C) Detection of cytochrome $c$ levels in H22 cells by western blot analysis subsequent to transfection with the pIRVP3IL-18HN plasmid. (D) Examination of caspase-3 activity in H22 cells by enzyme-linked immunosorbent assay at $72 \mathrm{~h}$ after pIRESneo and pIRVP3IL-18HN plasmid transfection revealed that casapase-3 activity was significantly increased in pIRVP3IL-18HN-transfected cells compared with pIRESneo-transfected cells. *P=0.018. GAPDH, glyceraldehyde 3-phosphate dehydrogenase.

the HN, VP3 and IL-18 genes in the pIRVP3IL-18HN plasmid markedly increased the inhibitory effects on $\mathrm{H} 22$ tumor volume compared with plasmids containing the single genes, and resulted in the lowest growth rate of the tumor (Fig. 3A and C). Compared with the empty plasmid treatment group, the pIRVP3IL-18HN recombinant plasmid carrying the HN, VP3 and IL-18 genes had a tumor inhibition rate of $46.28 \%$, whereas the pIRHN and pIRVP3 groups had inhibition rates of 26.57 and $31.36 \%$, respectively (Fig. 3B). Histopathological analysis was performed on biopsy specimens that were obtained from tumor-bearing C57BL/6 mice. Normal cancer cell morphology was observed, which also showed vigorous proliferation (Fig. 3Da and c). By contrast, in pIRVP3IL-18HN-transfected tumors, a vast majority of the cancer cells exhibited vacuoles and only in certain areas were there non-viable cells (Fig. 3Da and d). Additional ultrastructural analysis of tumor tissues revealed normal structure of the tumorigenic nuclei, with evenly distributed chromatin in the PBS group (Fig. 3Ea). However, in the pIRVP3IL-18HN group, nuclear shrinkage with chromatin margination (Fig. 3Eb), mitochondrial swelling, disappearance of the mitochondrial crista and lighter coloring by electron microscopic examination (Fig. 3Ec), as well as formation of typical apoptotic bodies (Fig. 3Ed), were observed.

\section{Discussion}

Combined gene therapy refers to strategies that utilize two or more genes simultaneously in the treatment of cancer (18). It combines complementary advantages of differential gene expression to potentiate the therapeutic effects. Certain studies have integrated 4 target genes, including B7-1, GM-CSF, p53 and $I L-2$, into a single adenoviral vector, which was imported into liver tumor cells and subsequently achieved satisfactory efficacy $(19,20)$. Su et al (21) also evaluated the synergistic effect of the $H S V$-tk and $I L-2$ genes in a liver cancer mouse model and confirmed that joint application of the $H S V$ - $t k$ and $I L-2$ genes had an improved therapeutic efficacy over gene therapy using any of the genes alone. In the present study, a recombinant DNA vaccine co-expressing the $N D V H N$ gene, chicken anemia virus $V P 3$ gene and $I L-18$ was constructed. 
A

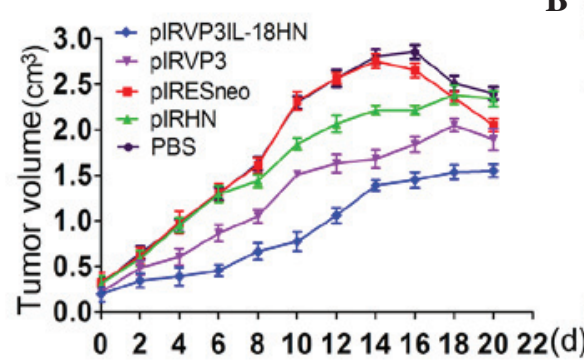

C

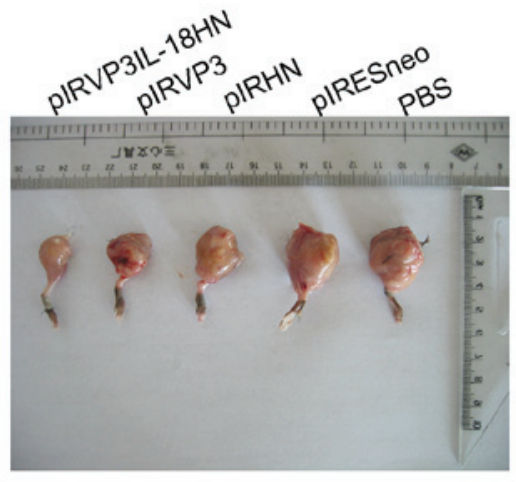

B

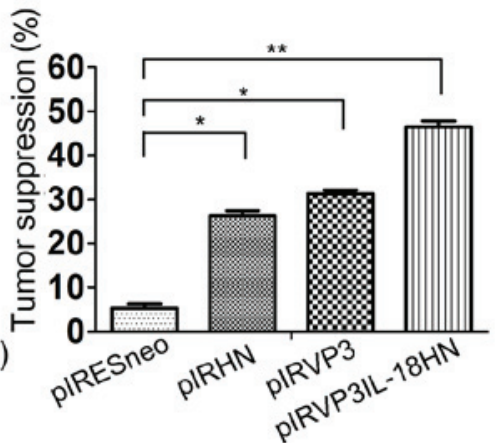

D

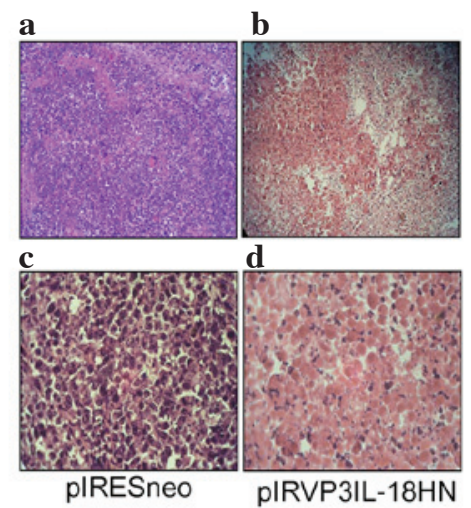

$\mathbf{E}$

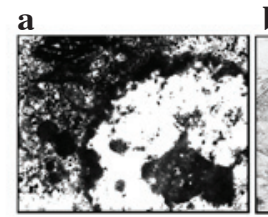

pIRESneo b

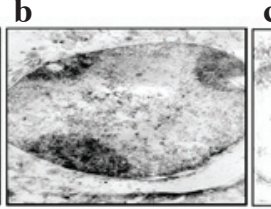

c

d

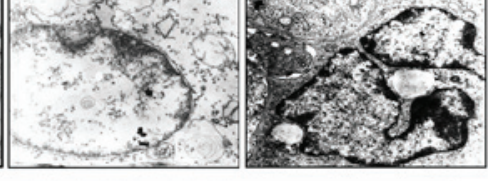

pIRVP3IL-18HN

Figure 3. Tumor suppressive effects of pIRVP3IL-18HN in vivo. (A) The growth curve of H22 tumors upon treatment with the recombinant plasmid carrying the HN, VP3 and IL-18 genes. (B) Comparison of tumor inhibition rates on treatment with various recombinant plasmids revealed that pIRVP3IL-18HN-transfection resulted in the slowest tumor growth rate. (C) Imaging of the femurs of H22 hepatoma-bearing mice. (D) Histopathological analysis of the tumor tissue biopsy following transfection with pIRESneo and pIRVP3IL-18HN using a light microscope at (Da and Db) x100 and (Dc and Dd) x400 magnification. (Ea) Normal structure of the tumorigenic nuclei in PBS group and (Eb) chromatin margination, (Ec) mitochondrial swelling and (Ed) apoptotic body formation subsequent to transfection with pIRVP3IL-18HN, as observed in tumor tissues under an electron microscope that was set at a x16,000 magnification. "P<0.05; ${ }^{* * *} \mathrm{P}<0.01$. PBS, phosphate-buffered saline.

The overall objective was to exploit the tumoricidal effects of the DNA vaccine and the synergistic function between expression of all three genes to reinforce tumor cell inhibition.

The development and progression of tumors is a highly complicated process involving comprehensive mechanisms, such as the mutation of the tumor supressor genes p53 and BRCA1 (22). Tumorigenesis is associated with abnormal proliferation, arrested differentiation and imbalanced apoptosis of tumor cells. Apoptosis in mammals engages the interplay of intricate mechanisms that are affected by multiple factors. There are three genes, consisting of $p 53, \mathrm{Bcl}-2$ and $c$-myc, that are considered the major apoptosis-associated genes (23-25). The occurrence and progression of tumors may be controlled when the tumor cells are induced to undergo apoptosis. Following a viral infection, the body will activate self-controlled genes to trigger programmed-cell death or apoptosis in order to maintain normal physiological activities and to minimize the damage caused by the virus, which ultimately leads to cell death $(26,27)$.
In addition, apoptosis may be regulated at the genetic level due to the expression of certain viral proteins or the triggering of novel genes $(28,29)$. In recent years, specifically infecting tumor cells with viruses to induce apoptosis became an important aspect of cancer biotherapy, thus further manipulating tumor development $(18,30)$. Apoptosis is the process of naturally occurring cell death under genetic regulation that also plays pivotal roles in the development of multicellular animals in terms of cytotoxicity (31), anti-viral activity, immune regulation, transcriptional regulation and other biological activities (32). The death of virus-infected cells would substantially limit viral replication and viral protein expression, thus ultimately eliminating the spread of the virus within the host. With additional research in cancer biology and comprehensive understanding of the complex associations between the tumor and the host, the rationale for cancer gene therapy may be expanded with an increasing number of potential targeted genes. The findings of the present study suggest that recombinant DNA vaccines containing the VP3, IL-18 and HN genes may be applied as a potential treatment for liver cancer. 


\section{Acknowledgements}

The present study was supported by National Natural Science Foundation of China (grant nos. 81225025 and 91229201) and Ministry of Health Foundation for State Key Clinical Department, 863 and 973 programs, in China (grant nos. 2012AA02A201 and 2013CB944903) .

\section{References}

1. Brand K, Löser P, Arnold W, Bartels T and Strauss M: Tumor cell-specific transgene expression prevents liver toxicity of the adeno-HSVtk/GCV approach. Gene Ther 5: 1363-1371, 1998.

2. Baqué P, Pierrefite-Carle V, Gavelli A, Brossette N, Benchimol D, Bourgeon A, Staccini P, Saint-Paul MC and Rossi B: Naked DNA injection for liver metastases treatment in rats. Hepatology 35 $1144-1152,2002$

3. Li Y, Li B, Li CJ and Li LJ: Key points of basic theories and clinical practice in rAd-p53 $\left(\right.$ Gendicine $\left.^{\mathrm{TM}}\right)$ gene therapy for solid malignant tumors. Expert Opin Biol Ther 15: 437-454, 2015.

4. Baliaka A, Zarogoulidis P, Domvri K, Hohenforst-Schmidt W, Sakkas A, Huang H, Le Pivert P, Koliakos G, Koliakou E, Kouzi-Koliakos $\mathrm{K}$, et al: Intratumoral gene therapy versus intravenous gene therapy for distant metastasis control with 2-diethylaminoethyl-dextran methyl methacrylate copolymer non-viral vector-p53. Gene Ther 21: 158-167, 2014.

5. Niu HX, Du T, Xu ZF, Zhang XK and Wang RG: Role of wild type p53 and double suicide genes in interventional therapy of liver cancer in rabbits. Acta Cir Bras 27: 522-528, 2012.

6. Fargnoli AS, Katz MG, Williams RD, Margulies KB and Bridges CR: A needleless liquid jet injection delivery method for cardiac gene therapy: A comparative evaluation versus standard routes of delivery reveals enhanced therapeutic retention and cardiac specific gene expression. J Cardiovasc Transl Res 7: 756-767, 2014.

7. Wang J, Wang C, Feng N, Wang H, Zheng X, Yang S, Gao Y, Xia X, Yin R, Liu X, et al: Development of a reverse genetics system based on RNA polymerase II for Newcastle disease virus genotype VII. Virus Genes 50: 152-155, 2015.

8. Alexander DJ: Newcastle disease and other avian paramyxoviruses. Rev Sci Tech 19: 443-462, 2000.

9. Zeng J, Fournier P and Schirrmacher V: Induction of interferon-alpha and tumor necrosis factor-related apoptosis-inducing ligand in human blood mononuclear cells by hemagglutinin-neuraminidase but not $\mathrm{F}$ protein of Newcastle disease virus. Virology 297: 19-30, 2002.

10. Sui H, Bai Y, Wang K, Li X, Song C, Fu F, Zhang Y and Li L: The anti-tumor effect of Newcastle disease virus HN protein is influenced by differential subcellular targeting. Cancer Immunol Immunother 59: 989-999, 2010.

11. Rajmani RS, Singh PK, Ravi Kumar G, Saxena S, Singh LV, Kumar R, Sahoo AP, Gupta SK, Chaturvedi U and Tiwari AK: In-vitro characterization and evaluation of apoptotic potential of bicistronic plasmid encoding $\mathrm{HN}$ gene of Newcastle disease virus and human TNF- $\alpha$. Anim Biotechnol 26: 112-119, 2015.

12. Brenu EW, van Driel ML, Staines DR, Ashton KJ, Ramos SB, Keane J, Klimas NG and Marshall-Gradisnik SM: Immunological abnormalities as potential biomarkers in chronic fatigue syndrome/myalgic encephalomyelitis. J Transl Med 9: 81, 2011.

13. Dong D, Gao J, Sun Y, Long Y, Li M, Zhang D, Gong J, Xu L, Li L, Qin S, et al: Adenovirus-mediated co-expression of the TRAIL and HN genes inhibits growth and induces apoptosis in Marek's disease tumor cell line MSB-1. Cancer Cell Int 15: 20, 2015.

14. Guan G, Jin N, Li X, Sun L, Jin C, Lou W, Shi P and Hao Y: Anti-tumor effects on human laryngeal carcinoma Hep-2 of recombinant fowlpox virus expressing chicken anemia virus Apoptin gene. Lin Chung Er Bi Yan Hou Tou Jing Wai Ke Za Zhi 23: 264-266, 2009 (In Chinese).

15. Natesan S, Kataria JM, Dhama K, Bhardwaj N and Sylvester A: Anti-neoplastic effect of chicken anemia virus VP3 protein (apoptin) in Rous sarcoma virus-induced tumours in chicken. J Gen Virol 87: 2933-2940, 2006.
16. Du G, Ye L, Zhang G, Dong Q, Liu K and Tian J: Human IL18-IL2 fusion protein as a potential antitumor reagent by enhancing NK cell cytotoxicity and IFN- $\gamma$ production. J Cancer Res Clin Oncol 138: 1727-1736, 2012.

17. National Research Council (US) Institute for Laboratory Animal Research: Guidance for the Description of Animal Research in Scientific Publications. National Academies Press, Washington DC, 2011.

18. Witlox MA, Lamfers ML, Wuisman PI, Curiel DT and Siegal GP: Evolving gene therapy approaches for osteosarcoma using viral vectors: Review. Bone 40: 797-812, 2007.

19. Pan D, Wei X, Liu M, Feng S, Tian X, Feng X and Zhang X: Adenovirus mediated transfer of p53, GM-CSF and B7-1 suppresses growth and enhances immunogenicity of glioma cells. Neurol Res 32: 502-509, 2010.

20. Shimizu T, Shimada H, Ochiai T and Hamada H: Enhanced growth suppression in esophageal carcinoma cells using adenovirus-mediated fusion gene transfer (uracil phosphoribosyl transferase and herpes simplex virus thymidne kinase). Cancer Gene Ther 8: 512-521, 2001.

21. Su H, Lu R, Ding R and Kan YW: Adeno-associated viral-mediated gene transfer to hepatoma: Thymidine kinase/interleukin 2 is more effective in tumor killing in non-ganciclovir (GCV)-treated than in GCV-treated animals. Mol Ther 1: 509-515, 2000.

22. Cai BH, Chen JY, Lu MH, Chang LT, Lin HC, Chang YM and Chao CF: Functional four-base A/T gap core sequence CATTAG of P53 response elements specifically bound tetrameric P53 differently than two-base A/T gap core sequence CATG bound both dimeric and tetrameric P53. Nucleic Acids Res 37: 1984-1990, 2009.

23. Knoll S, Fürst K, Thomas S, Villanueva Baselga S, Stoll A, Schaefer S and Pützer BM: Dissection of cell context-dependent interactions between $\mathrm{HBx}$ and 553 family members in regulation of apoptosis: A role for HBV-induced HCC. Cell Cycle 10: 3554-3565, 2011.

24. Barneda-Zahonero B, Collazo O, Azagra A, Fernández-Duran I, Serra-Musach J, Islam AB, Vega-García N, Malatesta R, Camós M, Gómez A, et al: The transcriptional repressor HDAC7 promotes apoptosis and c-Myc downregulation in particular types of leukemia and lymphoma. Cell Death Dis 6: e1635, 2015.

25. Cao Y, Yang G, Hunter ZR, Liu X, Xu L, Chen J, Tsakmaklis N, Hatjiharissi E, Kanan S, Davids MS, et al: The BCL2 antagonist ABT-199 triggers apoptosis, and augments ibrutinib and idelalisib mediated cytotoxicity in CXCR4 Wild-type and CXCR4 WHIM mutated Waldenstrom macroglobulinaemia cells. Br J Haematol 170: 134-138, 2015.

26. Moon J, Koh SS, Malilas W, Cho IR, Kaewpiboon C, Kaowinn S, Lee K, Jhun BH, Choi YW and Chung YH: Acetylshikonin induces apoptosis of hepatitis B virus $\mathrm{X}$ protein-expressing human hepatocellular carcinoma cells via endoplasmic reticulum stress. Eur J Pharmacol 735: 132-140, 2014.

27. Yang JJ, Zhang Y, Deng XZ, Xu K, Wang ZC, Wang J, Feng L and Ding WL: Influence of $F$ protein of hepatitis $C$ virus subtype $1 \mathrm{~b}$ inhibits on human hepatocellular carcinoma HepG2 cell apoptosis. Zhonghua Liu Xing Bing Xue Za Zhi 30: 388-392, 2009.

28. Follstaedt SC, Barber SA and Zink MC: Mechanisms of minocycline-induced suppression of simian immunodeficiency virus encephalitis: Inhibition of apoptosis signal-regulating kinase 1. J Neurovirol 14: 376-388, 2008.

29. Son KN and Lipton HL: Inhibition of Theiler's virus-induced apoptosis in infected murine macrophages results in necroptosis. Virus Res 195: 177-182, 2015.

30. Kim ES, Khuri FR and Herbst RS: Epidermal growth factor receptor biology (IMC-C225). Curr Opin Oncol 13: 506-513, 2001.

31. Lee SH, Kim MJ, Kim DW, Kang CD and Kim SH: Amurensin $\mathrm{G}$ enhances the susceptibility to tumor necrosis factor-related apoptosis-inducing ligand-mediated cytotoxicity of cancer stem-like cells of HCT-15 cells. Cancer Sci 104: 1632-1639, 2013.

32. Youn YS, Shin MJ, Chae SY, Jin CH, Kim TH and Lee KC: Biological and physicochemical evaluation of the conformational stability of tumor necrosis factor-related apoptosis-inducing ligand (TRAIL). Biotechnol Lett 29: 713-721, 2007. 